\title{
Influence of Dislocations in Thomson's Problem
}

\author{
A. Pérez-Garrido ${ }^{a}$, M. J. W. Dodgson ${ }^{b, c}$ and M. A. Moore ${ }^{b}$ \\ a Departamento de Física, Universidad de Murcia, Murcia 30.071, Spain \\ ${ }^{b}$ Theoretical Physics Group, Department of Physics and Astronomy, \\ The University of Manchester, M13 9PL, UK \\ c Theoretische Physik, ETH-Hönggerberg, CH-8093 Zürich, Switzerland
}

\begin{abstract}
We investigate Thomson's problem of charges on a sphere as an example of a system with complex interactions. Assuming certain symmetries we can work with a larger number of charges than before. We found that, when the number of charges is large enough, the lowest energy states are not those with the highest symmetry. As predicted previously by Dodgson and Moore, the complex patterns in these states involve dislocation defects which screen the strains of the twelve disclinations required to satisfy Euler's theorem.
\end{abstract}

Typeset using REVTEX 


\section{INTRODUCTION}

The properties of real systems are often determined by a large number of interacting entities. Progress in understanding such systems relies on the astute combination of analytical and numerical tools. This paper presents numerical studies on Thomson's problem for numbers of particles much larger than have been studied in the past. Thomson's problem is to find the lowest energy of $N$ Coulomb charges distributed on the surface of a unit sphere. Although the problem is simple to state, its solution is non-trivial, and numerical means are required to treat the interplay of frustration with ordering [1] 团. On a local scale, the charges are best distributed as a triangular lattice with six neighbours to each charge. Over the whole sphere, however, Euler's theorem dictates that there must exist twelve disclinations - charges with only five nearest neighbours.

The problem of constructing a lattice-like structure over a spherical surface has attracted attention in other contexts. A model with a spherical geometry of a two-dimensional electron system in a perpendicular magnetic field has been used to study the quantum Hall effect [5] and vortices in a thin-film superconductor [6 8]. The magnetic field is due to a Dirac monopole at the centre of the sphere. Using numerical work on this model for vortices, along with the continuum elasticity theory of topological defects on a curved surface (see Ref [9]), it was claimed in Ref [8] that including additional defects to the necessary twelve

disclinations could lower the energy of configurations on the sphere. These defects are dislocations (essentially bound pairs of fivefold and sevenfold disclinations) with a particular orientation. This improved on the work of Ref [7] where only the twelve disclinations were included, with the result that the energy per particle of a lattice on a sphere was greater than for a flat plane even in the thermodynamic limit of $N \rightarrow \infty$, because of the long range strains associated with the disclinations. The inclusion of dislocations allows the same thermodynamic limit to be recovered, as they may screen these long range strains.

The continuum elasticity theory allows the number of degrees of freedom to be reduced from the total number of particles to the number of disclination and dislocation defects. 
Following Ref [8], if we consider the twelve disclinations to be fixed at the corners of an icosahedron, the total elastic energy $E_{\text {tot }}$ of the system can be written as

$$
E_{\text {tot }}=E_{12}+N_{d} E_{d}+\sum_{s=1}^{12} \sum_{d=1}^{N_{d}} E_{s d}+\sum_{d=1}^{N_{d}} \sum_{d^{\prime}>d}^{N_{d}} E_{d^{\prime} d},
$$

where $E_{12}$ is the interaction and self energy of the twelve disclinations, $E_{d}$ is the self energy of a dislocation and the disclination-dislocation and dislocation-dislocation pairwise interactions are given by $E_{s d}$ and $E_{d^{\prime} d}$ respectively. These energies vary in a non-trivial manner with the ratio $R / l_{0} \propto N^{1 / 2}$, where $R$ is the radius of the sphere and $l_{0}$ is the mean lattice spacing. Summing up the results of [B], $E_{s d}$ increases more rapidly with $N$ than the self energy and the dislocation-dislocation energy. Depending on the orientation, it may also be negative so that dislocations could lower the total energy of the system. The energy landscape of a single dislocation has minima along the lines that join the disclinations.

The values of $N$ with the most stable ground states are those which are compatible with icosahedral symmetry [7]. The structures of proteins (capsomeres) in the shells (capsids) of spherical viruses are known to display icosahedral symmetry [10], and it was in this context that these compatible numbers were predicted. Each possible structure is defined by the translations along the triangular lattice vectors between each disclination $(h f, k f)$, which gives a "magic" number of $N=10 P f+2$ particles where $P=h^{2}+k^{2}+h k$ ( $h$ and $k$ are integers without common factors, $f$ is an integer, and $P f$ is the triangulation number denoted by $T$ in the literature) [11,12]. The same numbers and structures observed in nature (in particular $N=72$ and 132) were found in the numerical work of Ref [7], and are also found in Thomson's problem.

As Thomson's problem involves a simpler interaction than the model of vortices over a sphere, we have chosen it for numerical studies of ground states on a sphere with large $N$. Both the numerical and analytical results of Ref [8] suggested that sizes with $N$ of order $10^{3}$ must be reached before the dislocations will be energetically favourable. This explains why they have not been seen in previous work on Thomson's problem, which has concentrated on finding the ground states for smaller system sizes to very high accuracy. We will not be 
so concerned with locating the absolute ground state, but rather we will look for the general features of the low energy configurations, especially the influence of dislocations on the total energy.

\section{NUMERICAL METHOD}

To treat systems of charges with $N \sim 10^{3}$, a different approach is developed from earlier work on Thomson's problem. The highest number used in this problem before was $N=200$ using a genetic algorithm [四]. To reduce the number of degrees of freedom, we imposed certain symmetries on the system: fivefold rotational symmetry about a given axis and a twofold rotational symmetry about a perpendicular axis (these are symmetries possessed by an icosahedron). To do this, we first keep two charges fixed on the poles of the sphere. We then impose the symmetries by placing clusters of ten charges, represented by $\left\{\theta_{i}, \phi_{i}\right\}$, at the polar coordinates $\left(\theta_{i}, \phi_{i}+n \frac{2 \pi}{5}\right)$ and $\left(\pi-\theta_{i},-\phi_{i}+n \frac{2 \pi}{5}\right)$ for $n=0,1,2,3,4$.

The energy $E_{T}$ of the system is given by the relation

$$
E_{T}=E_{p}+\sum_{i}^{N_{c}} E_{p i}+\sum_{i}^{N_{c}} E_{i}+\sum_{i>j}^{N_{c}} E_{j i} .
$$

The first term is the potential energy of the two charges on the poles (always equal to $\frac{1}{2}$ for a unit sphere; the potential energy of two unit charges separated by a distance $d$ is given by $1 / d)$, the first sum is the potential energy of each cluster with the poles, the second sum is the potential energy of the charges in the same cluster and the last term is the energy of interaction of clusters with each other, where $N_{c}=(N-2) / 10$ is the number of clusters. This reduction of the degrees of freedom by approximately a factor of ten allows Thomson's problem to be tractable for thousands of charges.

It has been found numerically that the number of metastable states $M$ increases with $N$ as [13]

$$
M \approx .382 \times \exp (0.0497 N)
$$


although this number may be reduced somewhat by the restrictions of symmetry we have imposed. As with the number of charges we are considering, $M$ is extremely high, the numerical optimization routine with arbitrary initial conditions will only find metastable states, and give little information on the properties of the ground state. To avoid useless searching of configuration space, we distribute the charges in some strategic initial configurations before allowing them to relax.

In the relaxation process we employ an algorithm very similar to that used by Erber and Hockney [1] but we do not simply move the charges in the direction of the electrostatic force. Instead each charge is moved to $m$ new places around the original position which lie on a cone of angle angle $\alpha$ whose axis is the direction of the electrostatic force and the position with lowest energy is selected as the new position if its energy is lower than the original energy. If all of the $m$ places have a higher energy than the original the charge is not moved. We use $m=6$, having checked that the increase of this parameter does not change the results. When no charges can be moved, the angle $\alpha$ is decreased and the process is repeated. The program finishes when the energy reduction for every charge is less than a given number. Decreasing the size of that number increases the accuracy to which one knows the energy of the final state.

\section{RESULTS}

First, we have calculated the energy of the systems with 4002 and 5882 charges (which correspond to $T=400$ and $T=588$ ) with full icosahedral symmetry, (see Figure 1 for the case of 5882 charges). We then perturbed the initial configuration by displacing some charges on the line that join two disclinations. After relaxation, we found that the new states have less energy than those with full symmetry. We can observe the appearance of dislocations in Figure 2. To understand what is happening, note that charges away from dislocations are in a less strained environment than in the case of the icosahedral configuration.

We have also calculated the energy for $N=2132$ and 5792, with a special initial con- 
figuration. In this special configuration, we place the dislocations in a symmetric fashion. We put $5 p$ (p an integer) dislocations around each disclinations on the lines that join disclinations (i.e. we put a total of $12 \times 5 p$ dislocations, see figure 3 ) and let the system relax. With this initial configuration we obtain a new set of pseudo-magic numbers. We call them pseudo-magic as they may not have ground states of lower energy than other numbers, but they do have the possibility of arranging the dislocations symmetrically. In Figure 4 we show the final state obtained after relaxation. We see that with these system sizes, the disclination-dislocation interaction energy is not clearly dominant and the repulsion between dislocations is large enough to push the dislocations away from the line that joins disclinations. While the system was relaxing the charge configuration was observed, and we could see how dislocations moved over these lines. The patterns formed by the dislocations may be complex, but the results predicted by Dodgson and Moore [8] are seen to be emerging as $N$ increases. With larger systems we suspect that the dislocation-disclination energy will dominate so that the lines of dislocations will become straighter.

\section{CONCLUSIONS}

In this work, we found that the most symmetric states are not those with lowest energy in Thomson's problem for large system sizes. This is because the introduction of additional topological defects - dislocations - reduces the strain energy away from the disclinations, and the interactions between these defects allows for complex patterns. Our results should be valid for spherical systems with different interactions between the particles. For example, if spherical viruses exist with much larger numbers of protein units on the surface than has been found to date, similar patterns with dislocations should be present or the surface would be forced to deviate from that of a sphere. Dislocations in carbon nanotubes also play an important role as they determine their radius and helicity, and therefore control the electrical and mechanical properties of these new molecules [14, 15]. 


\section{ACKNOWLEDGEMENTS}

APG would like to acknowledge a grant and financial support from the Dirección General de Investigación Científica y Técnica, project number PB 93/1125. 


\section{REFERENCES}

[1] T. Erber and G. M. Hockney, J. Phys. A 24 L1369 (1991)

[2] E. L. Altschuler, T. J. Willians, E. R. Ratner, F. Dowla and F. Wooten, Phys. Rev. Lett. $722671(1994)$

[3] A. Pérez-Garrido, M. Ortuño, E. Cuevas and J. Ruiz, J. Phys. A 291973 (1996)

[4] J. R. Morris, D. M. Deaven and K. M. Ho, Phys. Rev. B 53 R1740 (1996)

[5] F. D. M. Haldane, Phys. Rev. Lett. 51605 (1983)

[6] J. A. O’Neill and M. A. Moore, Phys. Rev. Lett. 692582 (1992); Phys. Rev. B 48374 (1993); H. H. Lee and M. A. Moore, Phys. Rev. B 499240 (1995)

[7] M. J. W. Dodgson, J. Phys. A 292499 (1996)

[8] M. J. W. Dodgson and M. A. Moore, to be published in Phys. Rev. B (1997)

[9] H. S. Seung and D. R. Nelson, Phys. Rev. B 381005 (1988)

[10] C. J. Marzec and L. A. Day, Biophysical Journal 652559 (1993) and references therein

[11] D. L. D. Caspar and A. Klug, Cold Spring Harbor Symp. Quant. Biol. 271 (1962)

[12] D. L. D. Caspar, Phil. Trans. R. Soc. Lond. A 343133 (1993)

[13] T. Erber and G. M. Hockney, Phys. Rev. Lett. 741482 (1995)

[14] S. Iijima, Nature 35456 (1991)

[15] B. I. Dunlap, Phys. Rev. B 495643 (1994) 


\section{FIGURES}

FIG. 1. 5882 charges with full icosahedral symmetry. The disclinations, represented by larger spots, reside at the corners of an icosahedron. The energy is $E_{T}=17049766.73$.

FIG. 2. 5882 charges with dislocations. The dislocations are fivefold-sevenfold pairs and

the sevenfold centres are represented by the largest spots. The total energy is reduced to $E_{T}=17049653.24$.

FIG. 3. 5792 charges, initial symmetric configuration with dislocations. $E_{T}=16543582.87$.

FIG. 4. 5792 charges, final configuration. The more complex arrangement of dislocations results in a lower total energy of $E_{T}=16530072.09$. 
This figure "Fig1.gif" is available in "gif" format from: http://arxiv.org/ps/cond-mat/9701090v2 
This figure "Fig2.gif" is available in "gif" format from: http://arxiv.org/ps/cond-mat/9701090v2 
This figure "Fig3.gif" is available in "gif" format from: http://arxiv.org/ps/cond-mat/9701090v2 
This figure "Fig4.gif" is available in "gif" format from: http://arxiv.org/ps/cond-mat/9701090v2 